Article

\title{
Occurrence and Characterization of Fungi and Mycotoxins in Contaminated Medicinal Herbs
}

\author{
Ling Chen, Weipeng Guo, Yuqing Zheng, Jinzhen Zhou, Tingting Liu, Wei Chen, Daqing Liang, \\ Meiping Zhao, Yudan Zhu, Qingping Wu and Jumei Zhang *
}

State Key Laboratory of Applied Microbiology Southern China, Guangdong Provincial Key Laboratory of Microbial Culture Collection and Application, Guangdong Open Laboratory of Applied Microbiology, Guangdong Institute of Microbiology, Guangdong Academy of Sciences, Guangzhou 510070, China; angusy0662@hotmail.com (L.C.); guowp@gdim.cn (W.G.); zhengyuqing_work@hotmail.com (Y.Z.); zhoujz415@hotmail.com (J.Z.); LIUtt05@hotmail.com (T.L.); onlywei321@hotmail.com (W.C.); liangdaqing2019@hotmail.com (D.L.); zhaomeiping1992@hotmail.com (M.Z.); zhuyd19@gmail.com (Y.Z.); wuqp@gdim.cn (Q.W.)

* Correspondence: zhangjm@gdim.cn; Tel.: +86-20-3765-6160

Received: 1 November 2019; Accepted: 26 December 2019; Published: 3 January 2020

\begin{abstract}
Traditional medicinal herbs are widely used and may be contaminated with mycotoxigenic fungi during cultivation, harvesting, and storage, causing spoilage and mycotoxin production. We evaluated the predominant mycoflora and extent of mycotoxin contaminations in 48 contaminated samples of 13 different medicinal herbs. In total, $70.8 \%$ of herbs were slightly contaminated with aflatoxins $\left(<5 \mu \mathrm{g} \mathrm{kg}^{-1}\right)$. Codonopsis radix samples contained ochratoxin A (OTA) (360-515 $\mu \mathrm{g}$ $\left.\mathrm{kg}^{-1}\right)$, and Scutellariae radix samples contained OTA $\left(49-231 \mu \mathrm{g} \mathrm{kg}^{-1}\right)$ and citrinin $\left(15-53 \mu \mathrm{g} \mathrm{kg}^{-1}\right)$. Forty samples $(83.3 \%)$ contained fungal contamination. Sixty-nine strains were characterized via morphological and molecular identification. The predominant mycoflora comprised four genera, Aspergillus spp. (26.1\%), Penicillium spp. (24.6\%), Rhizopus spp. (14.5\%), and Trichoderma spp. (11.6\%). Aflatoxins, OTA, and citrinin were detected in 37 cultures by high-performance liquid chromatography-tandem mass spectrometry. Approximately 21.6\% of Aspergillus and Penicillium isolates produced mycotoxins. One Penicillium polonicum strain isolated from Scutellariae radix synthesized citrinin. Multiplex PCR analysis showed that three Aspergillus flavus strains harbored aflatoxin biosynthesis genes. One Aspergillus flavus strain isolated from Amomi fructus produced $\mathrm{AFB}_{1}$ and $\mathrm{AFB}_{2}$. To the best of our knowledge, the citrinin production by Aspergillus chevalieri and Penicillium sacculum was first reported in this study, which poses a potential risk of mycotoxin contamination in medicinal herbs.
\end{abstract}

Keywords: mycoflora; medicinal herbs; mycotoxin; contamination; aflatoxins; citrinin; mycotoxigenic fungi; multiplex PCR; HPLC-MS/MS

Key Contribution: This study systematically investigated the prevalence of fungi and mycotoxins in familiar contaminated medicinal herbs, Aspergillus chevalieri and Penicillium sacculum were first reported producing citrinin in medicinal herbs. Considering ochratoxin A was detected at high levels in Codonopsis radix and Scutellariae radix, it alludes to the need to establish a maximum permitted level for these medicinal herbs that are commonly consumed by humans.

\section{Introduction}

Chinese medicinal herbs are an invaluable asset to China, and widely used in medicinal, edible, and health products. With the continuous development of traditional medicinal herbs, the efficacy of 
medicinal herbs is recognized and valued, and its safety is a subject of concern. There have been many reports about the international trade obstacles of Chinese medicinal herbs in recent years, mainly on the case of excessive levels of extrinsic harmful residues in medicinal herbs including mycotoxins, pesticides, heavy metals, and sulfur dioxide [1-3].

Mildew is the primary cause of mycotoxin contamination among medicinal herbs. Medicinal herbs have been produced primarily through the traditional open, small workshop, and scattered planting business models. No uniform standard or efficient supervision method has been reported for processing, storage, and transportation of medicinal herbs. Mismanagement of medicinal herbs during processing, storage, and transportation and their intrinsic factors and external environmental conditions lead to severe spoilage and deterioration, along with mycotoxigenic fungi contamination. The majority of mycotoxin producers can be found in the fungal genera Aspergillus, Penicillium, Fusarium, and Alternaria which concomitantly happen to be the most abundant contaminants of food as well as medicinal herbs. The medicinal herbs (e.g., Notoginseng radix et rhizoma, Scutellariae radix, Morindae officinalis radix) are easily contaminated with molds producing mycotoxins such as ochratoxin A (OTA) [4]. Plant seeds (e.g., Coicis semen) are rich in starch, protein, and fat, which are highly susceptible to fungal contamination. The molds and mycotoxins contamination occur frequently in processing due to the heavily rainy days in harvest season as well as inappropriate storage and transportation of Coicis semen [5]. Mildew infestation in herbal medicines cause large-scale fungal growth and mycotoxin accumulation, thus affecting the quality and efficacy of medicinal herbs. More than 400 types of mycotoxins reportedly pose a risk to human health. Mycotoxins are secondary metabolites primarily produced by different fungal species. The mycotoxins contaminating medicinal herbs commonly include aflatoxins (AFs), OTA, and citrinin (CTN) [6]. Recent studies have found that Chinese herbal medicines can be simultaneously contaminated by various fungi, especially during storage [7]. The synergistic side effects of several mycotoxins on animal health and agricultural product performance are greater than those of individual mycotoxins [8]. For example, when OTA is used alone, the mortality rate is not high; however, it exerts synergistic effects with aflatoxins to increase the harmful effects of aflatoxins [9].

China is a large-scale producer of medicinal plant resources, with large-scale utilization of medicinal herbs [10]. Most medicinal herbs have a gradual and lasting effect. Some diseases require long-term use of medicinal herbs to achieve therapeutic outcomes. Certain medicinal herbs including Coicis semen and Jujubae fructus, which are used in medicines and food items, have also long been consumed as health foods. The long-term use of traditional medicinal herbs contaminated with mycotoxins may increase the incidence of adverse reactions [11]. Owing to the limited information available on toxigenic molds in medicinal herbs, this study aimed to evaluate the predominant mycoflora and the extent of fungal contamination in 13 different species of medicinal herbs (Jujubae fructus, Amomi fructus, Lycii fructus; Notoginseng radix et rhizoma, Codonopsis radix, Scutellariae radix, Morindae officinalis radix, Polygoni multiflori radix; Coicis semen; Poria; Ganoderma lucidum, Tremella fuciformis, Lentinus edodes) in common use from China, and to investigate their potential ability to produce mycotoxins from isolated fungi, such as aflatoxin $B_{1}\left(A F B_{1}\right)$, aflatoxin $B_{2}\left(A F B_{2}\right)$, aflatoxin $G_{1}\left(A F G_{1}\right)$, aflatoxin $\mathrm{G}_{2}\left(\mathrm{AFG}_{2}\right), \mathrm{CTN}$, and OTA.

\section{Results and Discussion}

\subsection{Mycotoxin Detection}

The high-performance liquid chromatography with fluorescence detection (HPLC-FLD) method (Figure S1) was optimized for the detection and quantification of six mycotoxins in medicinal herbs. The chromatogram, limit of quantification (LOQ), and limit of detection (LOD) are summarized in Figure $\mathrm{S} 1$ and Table S1. LOD values determined herein ranged from $0.012 \mu \mathrm{g} \mathrm{kg}^{-1}$ for $\mathrm{AFB}_{1}$ to $1.3 \mu \mathrm{g} \mathrm{kg}$ for CTN. Among 48 medicinal herbs, 39 (81.3\%) samples were positive for mycotoxins beyond their LODs (Table 1). Eight samples were simultaneously contaminated with two mycotoxins (AFB and 
OTA or CIN), and two samples were simultaneously contaminated with four mycotoxins $\left(\mathrm{AFB}_{1}, \mathrm{AFB}_{2}\right.$, $\mathrm{AFG}_{2}$, and OTA). In the case of medicinal plants, official regulations regarding the presence of only aflatoxins and OTA in medicinal herbs are shared globally among pharmacopoeias and national and organizational regulations. In general, the current legal limit for $\mathrm{AFB}_{1}$ in medicinal herbs ranges between $2 \mu \mathrm{g} \mathrm{kg}^{-1}$ and $10 \mu \mathrm{g} \mathrm{kg}^{-1}$, while the limit for total aflatoxins (combined $\mathrm{AFB}_{1}, \mathrm{AFB}_{2}, \mathrm{AFG}_{1}$, and $\mathrm{AFG}_{2}$ ) ranges from $4 \mu \mathrm{g} \mathrm{kg}^{-1}$ to $20 \mu \mathrm{g} \mathrm{kg}^{-1}$, and the limit for OTA rangs from $15 \mu \mathrm{g} \mathrm{kg}^{-1}$ to $80 \mu \mathrm{g}$ $\mathrm{kg}^{-1}$ [10]. Herein, $70.8 \%$ (34/48) of medicinal herbs were contaminated with $\mathrm{AFB}_{1}$; however their levels were within the permissible limits $\left(5 \mu \mathrm{g} \mathrm{kg}^{-1}\right)$. $\mathrm{AFB}_{1}$ and OTA were simultaneously detected in three varieties of Codonopsis radix OTA contamination being severe at $360-515 \mu \mathrm{g} \mathrm{kg}^{-1}$. Contaminant concentrations were higher than $1.2-158.7 \mu \mathrm{g} \mathrm{kg}^{-1}$ reported previously for 23 OTA-contaminated mold samples by Yang et al. [12]. OTA has been classified by the International Agency for Research on Cancer (IARC) as a potential carcinogen (group 2B) on the basis of sufficient animal studies [13]. The recommended provisional tolerable weekly intakes (PTWI) for OTA is $100 \mathrm{ng} \mathrm{kg}^{-1}$ body weight (bw), corresponding to approximately $14 \mathrm{ng} \mathrm{kg}^{-1} \mathrm{bw}$ for $1 \mathrm{~d}$ [14]. The Scientific Committee for Food has reported a tolerable daily intake (TDI) of $5 \mathrm{ng} \mathrm{kg}^{-1} \mathrm{bw}$ [15].

Table 1. Mycotoxin contamination in studied medicinal herbs.

\begin{tabular}{|c|c|c|c|c|c|c|c|}
\hline \multirow{2}{*}{ Scientific Name } & \multirow{2}{*}{$\begin{array}{l}\text { Sample } \\
\text { Name }\end{array}$} & \multicolumn{6}{|c|}{ Mean \pm SD $\left(\mu g \mathrm{~kg}^{-1}\right) *$} \\
\hline & & $\mathrm{AFB}_{1}$ & $\mathrm{AFB}_{2}$ & $\mathrm{AFG}_{1}$ & $\mathrm{AFG}_{2}$ & OTA & CTN \\
\hline \multirow[t]{3}{*}{$\begin{array}{l}\text { Notoginseng } \\
\text { radix et rhizoma }\end{array}$} & $02-1$ & $2.10 \pm 0.03$ & - & - & - & - & - \\
\hline & $02-2$ & $1.29 \pm 0.02$ & - & - & - & - & - \\
\hline & $02-3$ & $1.89 \pm 0.08$ & - & - & - & - & - \\
\hline \multirow[t]{3}{*}{ Codonopsis radix } & $03-1$ & $1.08 \pm 0.04$ & - & - & - & $420 \pm 5.99$ & - \\
\hline & $03-2$ & $0.89 \pm 0.02$ & - & - & - & $360 \pm 3.71$ & - \\
\hline & $03-3$ & $1.56 \pm 0.08$ & - & - & - & $515 \pm 9.23$ & $19 \pm 0.41$ \\
\hline \multirow[t]{3}{*}{ Scutellariae radix } & $04-1$ & $0.24 \pm 0.01$ & - & - & - & $49 \pm 1.74$ & - \\
\hline & 04-2 & - & - & - & - & $178 \pm 2.13$ & $53 \pm 0.98$ \\
\hline & $04-3$ & - & - & - & - & $231 \pm 3.44$ & $15 \pm 0.46$ \\
\hline \multirow[t]{3}{*}{$\begin{array}{c}\text { Morindae } \\
\text { officinalis radix }\end{array}$} & $05-1$ & $0.33 \pm 0.01$ & - & - & - & - & - \\
\hline & $05-2$ & $0.12 \pm 0.01$ & - & - & - & - & - \\
\hline & $05-3$ & $0.14 \pm 0.01$ & - & - & - & - & - \\
\hline \multirow[t]{7}{*}{$\begin{array}{c}\text { Ganoderma } \\
\text { lucidum }\end{array}$} & $06-1$ & $2.19 \pm 0.03$ & - & - & - & $0.79 \pm 0.02$ & - \\
\hline & $06-2$ & $1.80 \pm 0.12$ & - & - & - & $1.84 \pm 0.02$ & - \\
\hline & $06-3$ & $3.76 \pm 0.16$ & $0.43 \pm 0.003$ & - & $2.11 \pm 0.02$ & $0.48 \pm 0.03$ & - \\
\hline & $06-4$ & $1.81 \pm 0.07$ & $0.50 \pm 0.02$ & - & $0.87 \pm 0.02$ & $0.35 \pm 0.01$ & - \\
\hline & $06-5$ & $1.12 \pm 0.02$ & - & - & - & - & - \\
\hline & $06-6$ & $0.94 \pm 0.04$ & - & - & - & - & - \\
\hline & $06-7$ & $1.16 \pm 0.04$ & - & - & - & - & - \\
\hline \multirow[t]{3}{*}{ Coicis semen } & $07-1$ & $1.71 \pm 0.04$ & - & - & - & - & - \\
\hline & $07-2$ & $0.28 \pm 0.01$ & - & - & - & - & - \\
\hline & $07-3$ & $0.21 \pm 0.01$ & - & - & - & $0.81 \pm 0.01$ & - \\
\hline \multirow{6}{*}{$\begin{array}{c}\text { Amomi fructus } \\
\text { Tremella } \\
\text { fuciformis }\end{array}$} & $08-2$ & - & - & - & - & $11.4 \pm 0.28$ & - \\
\hline & $10-1$ & $0.72 \pm 0.02$ & - & - & - & - & - \\
\hline & $10-2$ & $0.80 \pm 0.02$ & - & - & - & - & - \\
\hline & $10-3$ & $3.05 \pm 0.09$ & - & - & - & - & $37 \pm 0.77$ \\
\hline & $10-4$ & $1.87 \pm 0.05$ & - & - & - & - & - \\
\hline & $10-5$ & $2.59 \pm 0.08$ & - & - & - & - & - \\
\hline \multirow[t]{3}{*}{ Lentinus edodes } & $11-1$ & $0.66 \pm 0.03$ & - & - & - & - & - \\
\hline & $11-2$ & $0.31 \pm 0.02$ & - & - & - & - & - \\
\hline & $11-3$ & $0.25 \pm 0.02$ & - & - & - & - & - \\
\hline
\end{tabular}


Table 1. Cont.

\begin{tabular}{|c|c|c|c|c|c|c|c|}
\hline \multirow{2}{*}{ Scientific Name } & \multirow{2}{*}{$\begin{array}{c}\text { Sample } \\
\text { Name }\end{array}$} & \multicolumn{6}{|c|}{ Mean \pm SD $\left(\mu \mathrm{g} \mathrm{kg}^{-1}\right) *$} \\
\hline & & $\mathrm{AFB}_{1}$ & $\mathrm{AFB}_{2}$ & $\mathrm{AFG}_{1}$ & $\mathrm{AFG}_{2}$ & OTA & CTN \\
\hline \multirow[t]{6}{*}{ Poria } & $12-1$ & $0.74 \pm 0.02$ & - & - & - & - & - \\
\hline & $12-2$ & $0.56 \pm 0.03$ & - & - & - & - & - \\
\hline & $12-3$ & $0.70 \pm 0.01$ & - & - & - & - & - \\
\hline & $12-4$ & $0.62 \pm 0.01$ & - & - & - & - & - \\
\hline & $12-5$ & $0.51 \pm 0.02$ & - & - & - & - & - \\
\hline & $12-6$ & $0.50 \pm 0.01$ & - & $0.85 \pm 0.02$ & - & - & - \\
\hline \multirow[t]{2}{*}{ Lycii fructus } & $13-1$ & - & - & - & - & $1.84 \pm 0.09$ & - \\
\hline & $13-2$ & - & - & - & - & $0.46 \pm 0.02$ & - \\
\hline
\end{tabular}

"-" below the limit of detection (LOD). Nine samples that were negative for mycotoxins below the LODs have not been shown in Table 1. The samples were Jujubae fructus (01-1;01-2; 01-3), Amomi fructus (08-1; 08-3), Polygoni multiflori radix (09-1; 09-2; 09-3), and Lycii fructus (13-3). "**" the mean of mycotoxins' production and standard deviation (SD).

Furthermore, $\mathrm{AFB}_{1}$ and $\mathrm{OTA}$ were simultaneously detected in one of three Scutellariae radix samples, OTA and CTN were detected in the other two samples. These OTA contamination levels (49-515 $\mu \mathrm{g} \mathrm{kg}^{-1}$ ) of Codonopsis radix and Scutellariae radix should not be disregarded. Considering the vulnerability of several medicinal herbs to OTA, we suggest that a maximum permitted level for this mycotoxin is urgently needed. Further studies are required to develop methods to prevent and control OTA contamination in Codonopsis radix and Scutellariae radix, including the identification of fungi causing OTA accumulation and establishment of detection standards for Codonopsis radix and Scutellariae radix to minimize human health risks.

\subsection{Fungal Contamination}

The association between fungal species and medicinal herbs remains unclear owing to complex sources of contamination including extrinsic (environmental and geographical) and intrinsic (constituents of each herbal species) factors [16]. Owing to the widespread presence of fungi in nature, medicinal herbs are often susceptible to fungal contamination inadequately treated during production and storage. The presence of fungi in 48 sample of 13 different medicinal herbs (each $\geq 3$ batches) were detected, results showed that $83.3 \%$ (40/48) were positive for fungi, demonstrating the majority of Chinese medicinal herbs were contaminated with fungi.

Sixty-nine fungi (64 strains of mold and 5 strains of yeast) were isolated from 48 medicinal herb samples. Preliminary morphological identification revealed 18 strains of Aspergillus, 17 strains of Penicillium, 10 strains of Rhizopus, 8 strains of Trichoderma, 4 strains of Mucor, 5 strains of yeast, 2 strains of Byssochlamys, 2 strains of Chaetomium, 1 strain of Alternaria, 1 strain of Mycosis, and 1 strain of Neurospora. The colony morphology of the primary strains are shown in Figure 1. Traditionally, fungi in medicinal herbs have been divided into two distinct classes: field fungi (e.g., Fusarium, Alternaria), which invade and produce their toxins before harvest; and storage fungi (e.g., Aspergillus, Penicillium), which become a problem after harvest [17]. Moreover, some fungi (e.g., Aspergillus) might belong to both classes before and after harvest. The predominant mycoflora isolated in this study were storage fungi. Particularly the primary isolated strains from Codonopsis radix and Scutellariae radix were Penicillium polonicum, which potentially produce CTN.

Mildewed medicinal herbs were markedly contaminated with fungi. Overall, Aspergillus and Penicillium were the most frequent contaminants, concurrent with previous findings $[18,19]$. Reproduction in Aspergillus spp. requires a substrate with a higher water activity, suggesting that the medicinal herbs herein may be not dried in time after harvesting, or that drying was gradual and the duration of dehydration was too long, resulting in subsequent mold propagation. The present drying procedure should be strengthened to reduce the possibility of mold contamination. Furthermore, herbal medicines are often affected with mildew during storage owing to poor management, especially in southern China. Currently, various methods can be used to reduce fungal 
contamination in medicinal herbs, primarily aiming to prevent, control, and degrade contaminants, i.e., prevention of fungal growth at the source to reduce in contaminated medicinal herbs. Special measures against mildew are required to establish a scientific and reasonable monitoring system, from the beginning of harvesting at the site of origin, with particular attention to the control of mold pollution, thus improving the production conditions of storage, processing, transportation, etc., and preventing subsequent contamination and mold propagation. However, once the mycotoxin is formed or exceeds the standard, subsequent mycotoxin degradation or detoxification methods are required to manage medicinal herbs. Microbial degradation of toxins has recently received increasing attention.

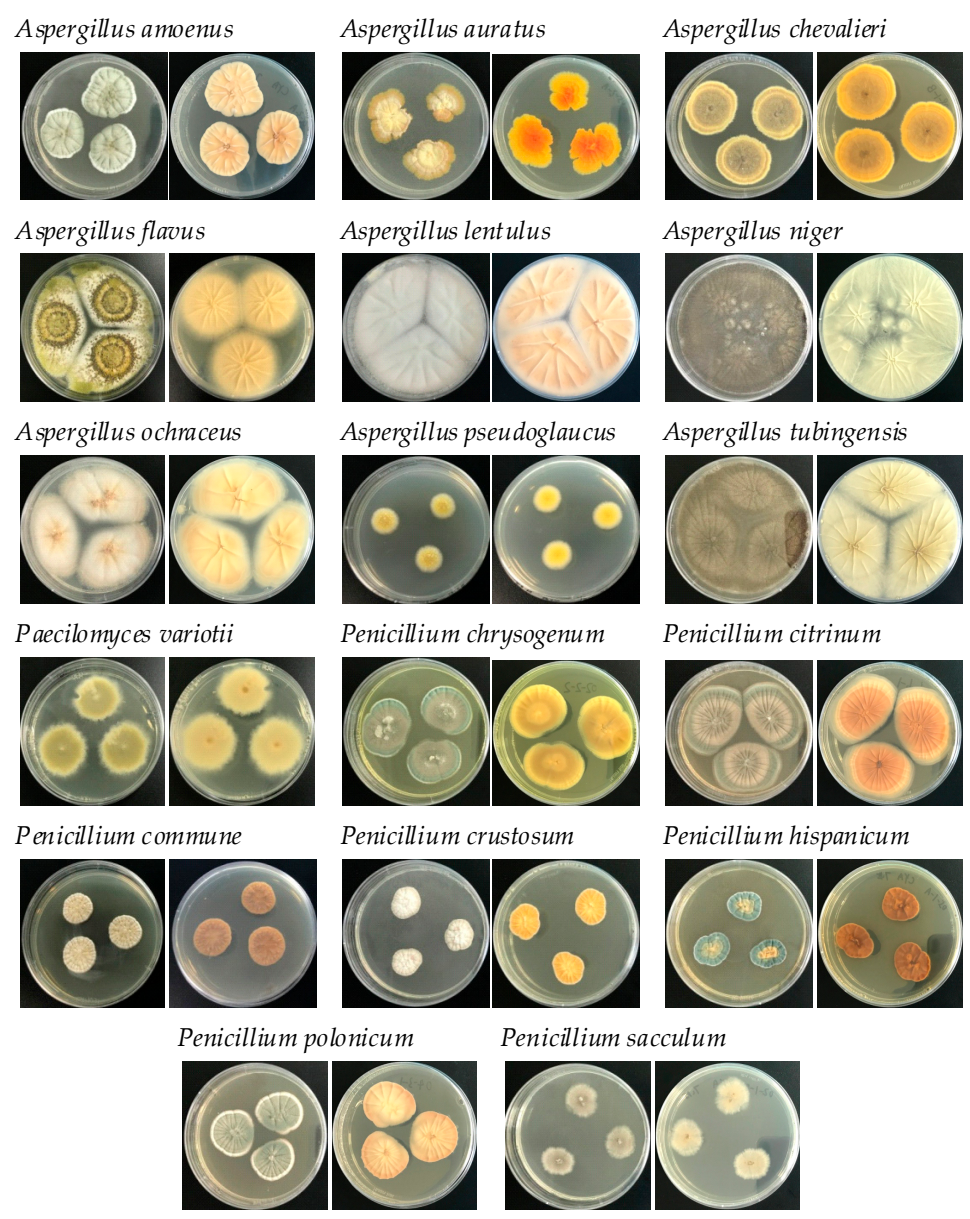

Figure 1. Colony morphology (top and reverse) of Aspergillus, Penicillium, and Paecilomyces species isolated in this study after $3-7 \mathrm{~d}$ of incubation at $25^{\circ} \mathrm{C}$ in the dark on Czapek Yeast Exatract Agar (CYA).

\subsection{Mycotoxigenic Potentials of the Fungal Isolates}

Six mycotoxins $\left(\mathrm{AFB}_{1}, \mathrm{AFB}_{2}, \mathrm{AFG}_{1}, \mathrm{AFG}_{2}, \mathrm{OTA}\right.$, and $\left.\mathrm{CTN}\right)$ in 37 strains isolated from 24 samples from 10 different types of medicinal herbs were determined qualitatively by HPLC-MS/MS method (Figure S1). Mycotoxins were detected positive in eight strains (Figure 2), e.g., $\mathrm{AFB}_{1}$ and $\mathrm{AFB}_{2} \mathrm{Were}$ simultaneously detected in one strain of Aspergillus flavus (from Amomi fructus 08-3), CTN was detected in one strain of Aspergillus chevalieri (from Amomi fructus 08-1), one strain of Aspergillus ochraceus (from Ganoderma lucidum 06-6), three strains of Penicillium citrinum (from Ganoderma lucidum 06-5, 06-6, 06-7), one strain of Penicillium polonicum (from Scutellariae radix 04-2), and one Penicillium sacculum (from Notoginseng radix et rhizoma 02-1). One strain of Penicillium polonicum isolated from Scutellariae radix produced CTN after culturing, and CTN was detected in Scutellariae radix; however, this is rare. OTA and CTN were detected in Codonopsis radix and Scutellariae radix; however, we could not isolate the fungi producing these mycotoxins. Medicinal herbs were speculated to be contaminated by 
mycotoxigenic fungi, and the mycotoxigenic fungi were eliminated in subsequent stages. Due to their wide range of physical and chemical properties, mycotoxins are stable chemical compounds which cannot be destroyed during most processing operations, the mycotoxins still existed in medicinal herbs. The fungi isolated herein were not original contaminated mycotoxigenic fungi. Phylogenetic analysis based on the ITS and $\beta$-tubulin gene of the 37 isolates, two reference strains, and other closely related species is showed in Figure 2.

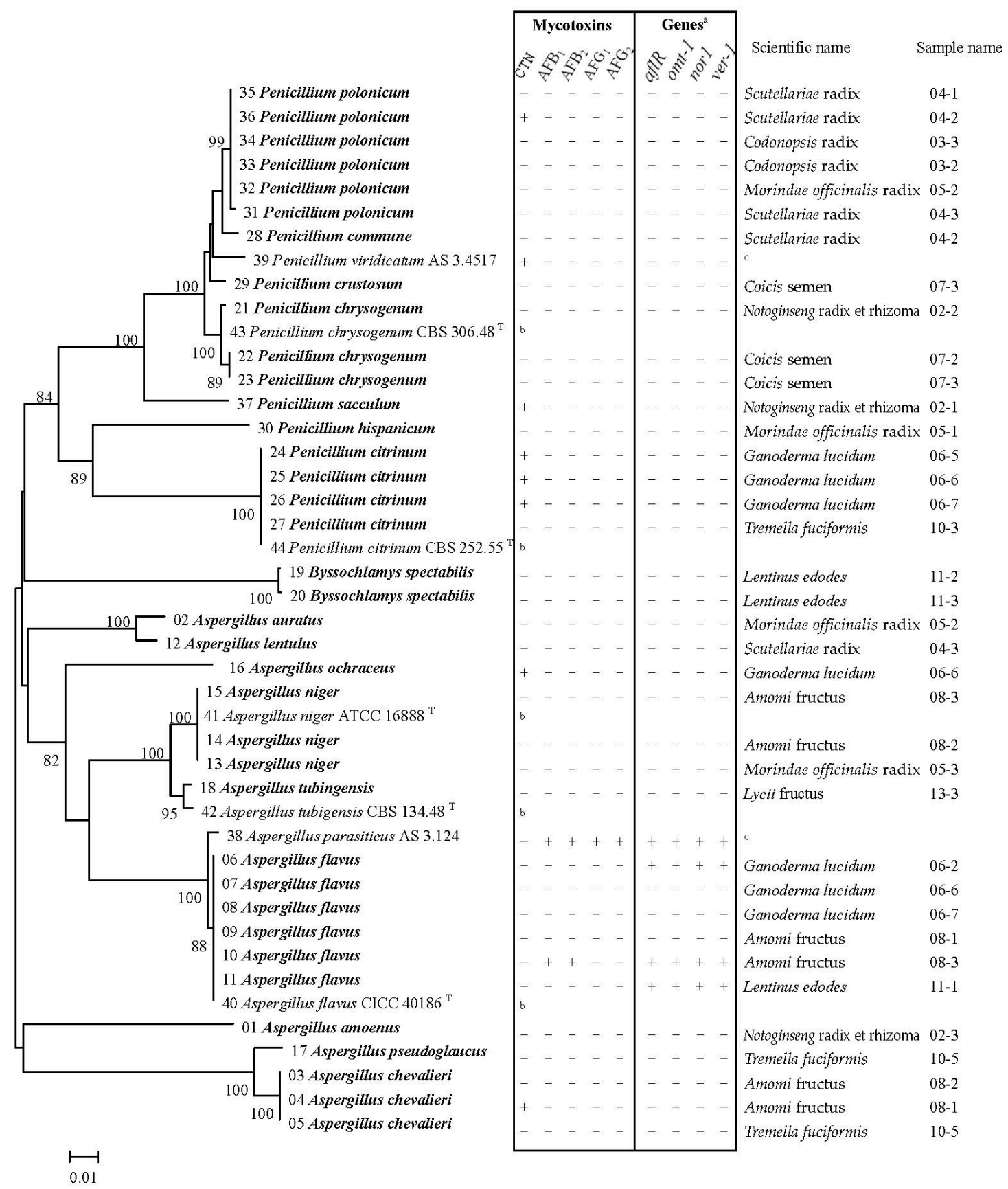

Figure 2. Neighbor-joining tree based on sequence data of ITS and $\beta$-tubulin from 44 strains using MEGA 7.0. Bootstrap values are shown in the nodes according to 1000 replications. Only bootstrap values $>80 \%$ are shown. Thirty-seven isolates from this study are shown in bold type. "a" aflatoxin biosynthesis genes; " $\mathrm{b}$ " untested; " $\mathrm{c}$ " standard strain used as positive control; " $\mathrm{T}$ " type strain; "+" detected; "-" not detected. 
To characterize mycotoxin-producing fungi via multiplex PCR, primers specific for structural and regulatory genes involved in AF biosynthesis (aflR1, omt-1, nor1, and ver-1) were assayed in Aspergillus and Penicillium (Table 2). Consequently, specific fragments amplified from the DNA of isolates with potential mycotoxin-producing abilities. Target genes were not amplified in certain Aspergillus strains. The probability of the production of a particular toxin may be predicted in accordance with the presence or absence of an amplification product; however, effective toxin biosynthesis remains unclear even through analytical chemistry analyses [19]. Thirty-nine strains, including 37 isolated from medicinal herbs and 2 standard strains, were assayed for genes involved in AF biosynthesis. Among the 37 strains, 3 displayed positive signals on using gene-based primer sets (Figure 2). Amplification products were obtained from three stains of Aspergillus flavus, thus indicating their potential for aflatoxin (AF) production. Only one strain of Aspergillus flavus (from Amomi fructus 08-3) produced $\mathrm{AFB}_{1}$ and $\mathrm{AFB}_{2}$ upon analytical chemistry analysis.

Table 2. Primer sets for genes involved in aflatoxin biosynthesis.

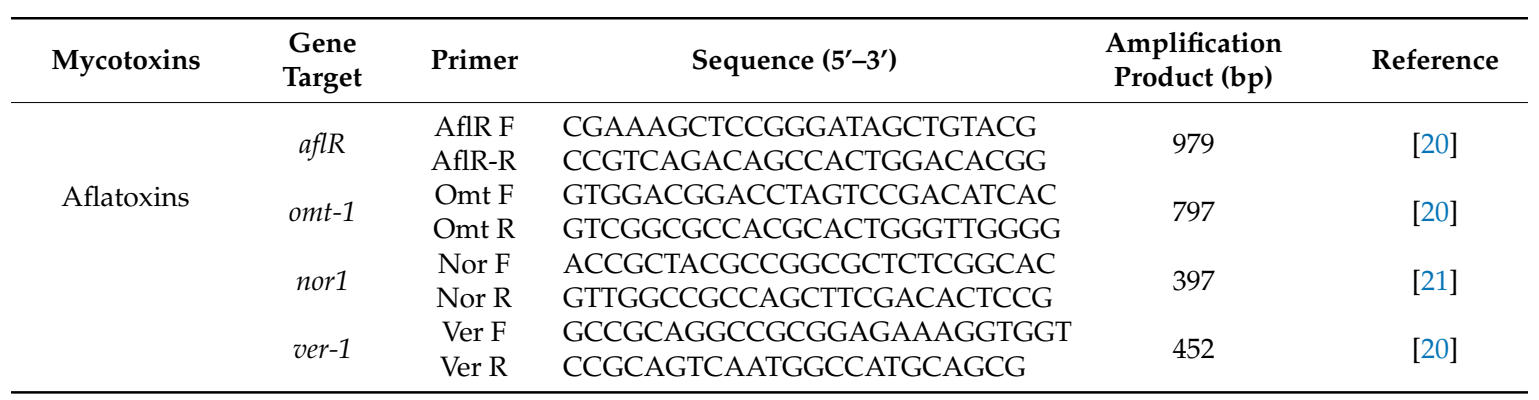

AF is a secondary metabolite produced by Aspergillus flavus and Aspergillus parasiticus and is a derivative of a class of dihydrofuran coumarin. The toxins isolated herein include $\mathrm{AFB}_{1}, \mathrm{AFB}_{2}, \mathrm{AFG}_{1}$, and $\mathrm{AFG}_{2}$, the most toxic one being $\mathrm{AFB}_{1}$. In 1993, the International Agency for Research on Cancer reported $A_{F B}$ as a Class 1 carcinogen [13], since it is 68-fold more toxic than arsenic, second only to botulinum, and is currently the most toxic mycotoxin. The 2015 edition of the Chinese Pharmacopoeia has specified 14 types of medicinal materials including Nelumbinis semen, Myristicae semen, and Jujubae fructus, and five types of animal medicinal materials such as Hirudo to detect AFs, and established a permissible limit of $10 \mathrm{\mu g} \mathrm{kg}^{-1}$ in these medicinal material [10]. Approximate 70.8\% (34/48) of medicinal herbs in this study were slightly contaminated with aflatoxins ( $<5 \mu \mathrm{g} / \mathrm{kg})$. Although 18 strains of Aspergillus have been isolated from medicinal herbs, only one culture of Aspergillus flavus isolated from Amomi fructus produces $\mathrm{AFB}_{1}$ and $\mathrm{AFB}_{2}$. The reason for this observation may be that Aspergillus strains did not harbored aflatoxin biosynthesis genes. Other causes of non-toxicity potentially include environmental factors (temperature, humidity, etc.) or other factors (such as different media). The specific reasons warrant further investigation. Amomi fructus may be a suitable substrate for Aspergillus flavus produced aflatoxin. More medicinal herbs such as Amomi fructus should be included to focus on mycotoxin monitoring.

CTN is a secondary metabolite in filamentous fungi in Penicillium, Aspergillus, and Monascus [22]. Penicillium is a toxin-producing genus, primarily including Penicillium citrinum, Penicillium cyclopium, Penicillium patulum, Penicillium urticae, and Penicillium viridicatum. These fungi potentially produce secondary metabolites such as CTN, cyclopiazonic acid, and patulin (PAT). CTN is another mycotoxin that has attracted increasing attention after AF. CTN primarily targets the kidney. CTN exerts cytotoxic and genotoxic effects in both in vivo and in vitro systems. It can cause kidney enlargement, increased urine output, tubular dilatation, and degeneration and necrosis of epithelial cells [23]. However, the molecular mechanisms underlying CTN-mediated biological functions and cytotoxicity remain unknown [24]. CTN and OTA are important mycotoxins, which often coexist in food and feed stuff. Gong [25] assessed the toxicity of OTA and CTN were individually and combinatorially in human embryonic kidney (HEK) 293 cells via the MTT assay and reported synergistic cytotoxic effects 
upon co-treatment with OTA and CTN, as revealed through significant accumulation of HEK293 cells in the $S$ and G2/M stages. Six strains of Penicillium polonicum were isolated, and only one strain from Scutellariae radix produced CTN. Three strains of Penicillium citrinum were isolated from Ganoderma lucidum, and all pure cultures produced CTN. Furthermore, a strain of Aspergillus ochraceus isolated from Ganoderma lucidum produced CTN. Ganoderma lucidum is a traditional Chinese medicinal herb, and polysaccharides are important functional components in the fungus. Over the years, we have performed in-depth studies on Ganoderma lucidum and its active substances. The effective development and utilization of Ganoderma lucidum resources has broad commercial prospects for human health. Monitoring of molds and mycotoxins has been recommended for Ganoderma lucidum to ensure the safety of Ganoderma lucidum and Ganoderma lucidum products.

CTN was detected in one strain of Aspergillus chevalieri isolated from Amomi fructus for the first time. Ismail et al. [26] reported that $\mathrm{AFB}_{1}, \mathrm{AFG}_{1}$, and Gliotoxin were produced in the extract of Eurotium chevalieri var. intermedium (=Aspergillus chevalieri var. intermedium). Among 95 strains of Eurotium assayed, CTN was detected in the extract of only one strain of Eurotium pseudoglaucum (=Aspergillus pseudoglaucum). The melting point of CTN is $175{ }^{\circ} \mathrm{C}$ Thus far, few studies have focused on CTN degeneration. Temperature and humidity markedly influence CTN degradation and detoxification. When CTN is heated to $175^{\circ} \mathrm{C}$ in a dry environment, it is applied to human cervical cancer cells and does not exert cytotoxic effects. At medium humidity, CTN is degraded and detoxified at $140{ }^{\circ} \mathrm{C}$ Therefore, high temperature treatment reduces the CTN content in food, thus reducing CTN intake. Degradation of toxins has recently received increasing attention.

\section{Conclusions}

This study systematically investigated the prevalence of fungi and mycotoxins in medicinal herbs. A relatively large number of medicinal herbs were found to be contaminated, mainly by Aspergillus and Penicillium. OTA was detected at high levels in Codonopsis radix and Scutellariae radix. CTN was detected in Scutellariae radix at $53 \mu \mathrm{g} \mathrm{kg}^{-1}$. Subsequent analysis indicates that Penicillium polonicum was the potential contributors to the high levels of CTN contamination in Scutellariae radix. Three strains of Penicillium citrinum isolated from Ganoderma lucidum can produce CTN. The presence of myco-fungi implies the potential for mycotoxin contamination under suitable conditions, and once the mycotoxins is formed or exceeds the standard, thus could pose a potential health risk to consumers. Considering the toxicological effects of OTA and CTN, a maximum permitted level in several medicinal herbs is needed. Furthermore, these data may help to identify the species of medicinal herbs that are more commonly associated with fungi and mycotoxin contaminations, and to provide useful information for effective prevent, control, and degrade contaminants strategies to ensure the safety of medicinal herbs.

\section{Materials and Methods}

\subsection{Reagents and Apparatus}

All solvents were of high-performance liquid chromatography (HPLC) grade and were purchased from CNW (ANPEL, Shanghai, China). Standards of aflatoxins (AFB 1 : $1.0 \mathrm{mg} / \mathrm{L}, \mathrm{AFB}_{2}: 1.0 \mathrm{mg} / \mathrm{L}$, $\left.\mathrm{AFG}_{1}: 0.3 \mathrm{mg} / \mathrm{L}, \mathrm{AFG}_{2}: 0.3 \mathrm{mg} / \mathrm{L}\right), \mathrm{CTN}$ (>98\%) and OTA (>99\%) were purchased from Pribolab (Immunos, Singapore) and o2si (Charleston, SC, USA).

HPLC analysis was performed with LC-20AT (Shmadzu Corp., Kyoto, Japan). HPLC-MS/MS analysis was performed with a 1290 Infinity Liquid Chromatograph interfaced to a 6430 Triple Quad Mass Spectrometer system.

MycoSep 226 aflaZon+ multifunctional column were obtained from Romer (Union, MO, USA). Immunoaffinity columns for total aflatoxins $\left(\mathrm{AFB}_{1}, \mathrm{AFB}_{2}, \mathrm{AFG}_{1}, \mathrm{AFG}_{2}\right)$ and Ochratoxin $\mathrm{A}$ were obtained from Beacon (Saco, ME, USA). Immunoaffinity columns for citrinin (CitriTest HPLC) were 
obtained from Vicam (Watertown, MA, USA). Milli-Q water was prepared in our laboratory using the Academic System (Millipore, Molsheim, France).

\subsection{Sampling}

Forty-eight samples of medicinal herbs, comprising 13 different species, were chosen for this study as it is susceptible to fungal growth and mycotoxins production. All samples (Table 3) purchased from the herbal market in China and immediately placed in a sterile polythene bag, properly sealed, and transported to the laboratory and immediately processed to prevent secondary contamination. The medicinal herbs were identified by Diling Chen (Associate professor, Senior postdoctoral researcher, Doctor of Medicine, Guangzhou University of Chinese Medicine, Guangdong Institute of Microbiology, Guangzhou, China) according to Pharmacopoeia of the People's Republic of China (Edition 2015) [27].

Table 3. Thirteen different species of medicinal herbs from China.

\begin{tabular}{cccc}
\hline Scientific Name & No. of Samples & Sample Name & Producing Regions \\
\hline Jujubae fructus & 3 & $01-1 ; 01-2 ; 01-3$ & Hebei \\
Notoginseng radix et & 3 & $02-1 ; 02-2 ; 02-3$ & Yunnan \\
rhizoma & 3 & $03-1 ; 03-2 ; 03-3$ & Gansu \\
Codonopsis radix & 3 & $04-1 ; 04-2 ; 04-3$ & Gansu \\
Scutellariae radix & 3 & $05-1 ; 05-2 ; 05-3$ & Guangdong \\
Morindae officinalis radix & 7 & $06-1 ; 06-2 ; 06-3 ; 06-4 ;$ & Shanxi \\
Ganoderma lucidum & 3 & $06-5 ; 06-6 ; 06-7$ & Fujian \\
Coicis semen & 3 & $07-1 ; 07-2 ; 07-3$ & Yunnan \\
Amomi fructus & 3 & $08-1 ; 08-2 ; 08-3$ & Guizhou \\
Polygoni multiflori radix & 5 & $09-1 ; 09-2 ; 09-3$ & Fujian \\
Tremella fuciformis & 3 & $10-1 ; 10-2 ; 10-3 ; 10-4 ; 10-5$ & Hubei \\
Lentinus edodes & & $11-1 ; 11-3$ & Zhejiang \\
Poria & 6 & $11-2 ;$ & Anhui \\
Lycii fructus & 3 & $12-1 ; 12-2 ; 12-3 ; 12-4 ;$ & Xinjiang \\
\hline
\end{tabular}

\subsection{Mycotoxin Analysis}

Production of six mycotoxins $\left(\mathrm{AFB}_{1}, \mathrm{AFB}_{2}, \mathrm{AFG}_{1}, \mathrm{AFG}_{2}, \mathrm{OTA}\right.$, and $\left.\mathrm{CTN}\right)$ were detected in accordance with the method of Pharmacopoeia of the People's Republic of China (Edition 2015) [27] and the national food safety standards of China [28-30]. The mycotoxins were quantified by comparing peak areas with a calibration curves obtained with standard solutions. Each standard curve comprised five different concentrations of reference solutions, thrice in parallel. All standard curves were linear and $R^{2}$ values were $>0.9998$. The sensitivity of the method was determined primarily on the basis of the limit of detection (LOD) and limit of quantification (LOQ). The LOD and LOQ of mycotoxins were calculated by considering the average noise signal and adding 3 and 10 standard deviations of noise, respectively. The results are shown in Table S1.

\subsection{1. $\mathrm{AFB}_{1}, \mathrm{AFB}_{2}, \mathrm{AFG}_{1}$, and $\mathrm{AFG}_{2}$ Prodution by Medicinal Herbs}

Five grams of samples was blended with $20 \mathrm{~mL}$ acetonitrile-water (84/16) and extracted using ultrasonography for $20 \mathrm{~min}$. After centrifugation at $6000 \mathrm{rpm}$ for $10 \mathrm{~min}$, the supernatant was purified and concentrated by MycoSep 226 aflaZon+ multifunctional column. $4 \mathrm{~mL}$ of the eluate were blow-dried at $50{ }^{\circ} \mathrm{C}$ with nitrogen, and the residue was dissolved in $200 \mu \mathrm{L}$ hexane and $100 \mu \mathrm{L}$ trifluoroacetic acid, vortex-mixed for $30 \mathrm{~s}$, derived in an incubator at $40^{\circ} \mathrm{C} \pm 1{ }^{\circ} \mathrm{C}$ for $15 \mathrm{~min}$. The derivative was blow-dried at $50^{\circ} \mathrm{C}$ with nitrogen, and the residue was dissolved in $1 \mathrm{~mL}$ of acetonitrile, vortex-mixed for $30 \mathrm{~s}$, passed through a $0.22-\mu \mathrm{m}$ microporous membrane, and harvested for injection. HPLC analysis was performed with LC-20AT coupled to a FLD detector set to $360 \mathrm{~nm}$ excitation and $440 \mathrm{~nm}$ emission. 
The sample was separated using a ZORBAX Eclipse Plus C18 column $(250 \times 4.6 \mathrm{~mm}$, 5-Micron, Agilent Technologies Co., Ltd., Santa Clara, CA, USA). The mobile phase consisted of (A) water and (B) acetonitrile, at a flow rate of $1.0 \mathrm{~mL} / \mathrm{min}$ in gradient mode, $0-13 \mathrm{~min}$ : $20 \%-30 \%$ of $\mathrm{B}, 13-17 \mathrm{~min}$ : $30 \%-40 \%$ of $\mathrm{B}, 17-18 \mathrm{~min}: 40 \%$ of $\mathrm{B}, 18-20 \mathrm{~min}: 40 \%-20 \%$ of $\mathrm{B}, 20-22 \mathrm{~min}: 20 \%$ of $\mathrm{B}$. The retention time of the $\mathrm{AFB}_{1}$ was about $11.7 \mathrm{~min}, 20.0 \mathrm{~min}$ for $\mathrm{AFB}_{2}, 9.5 \mathrm{~min}$ for $\mathrm{AFG}_{1}$ and $17.2 \mathrm{~min}$ for $A F G_{1}$.

\subsubsection{OTA Prodution by Medicinal Herbs}

Five grams of samples was blended with $20 \mathrm{~mL}$ methanol-water (80/20) and were placed for $30 \mathrm{~min}$ in a shaker, then filtered using quantitative filter paper. To $10 \mathrm{~mL}$ of extract, $40 \mathrm{~mL}$ of phosphate buffer solution (PBS, $8 \mathrm{~g} \mathrm{NaCl}, 1.2 \mathrm{~g} \mathrm{Na} \mathrm{HPO}_{4}, 0.2 \mathrm{~g} \mathrm{~K}_{2} \mathrm{HPO}_{4}, 0.2 \mathrm{~g} \mathrm{KCl}$, and brought up to $1 \mathrm{~L}$ with distilled water, $\mathrm{pH} 7.4 \pm 0.1$ ) was added, followed by glass fiber filter paper. The filtrate was purified by ochratoxin A immunoaffinity column (Beacon, $3 \mathrm{~mL}$ ). The immunoaffinity column was rinsed with $10 \mathrm{~mL}$ PBS buffer, $10 \mathrm{~mL}$ water and $1.5 \mathrm{~mL}$ of methanol in turn. The eluate was blow-dried at $45^{\circ} \mathrm{C}$ with nitrogen, and the residue was dissolved in $500 \mu \mathrm{g}$ of mobile phase. HPLC analysis was performed with LC-20AT coupled to a FLD detector set to $333 \mathrm{~nm}$ excitation and $460 \mathrm{~nm}$ emission. The sample was separated using a ZORBAX SB C18 column $(150 \times 4.6$ mm, 5-Micron, Agilent Technologies Co., Ltd., Santa Clara, CA, USA). The mobile phase was acetonitrile-water-glacial acetic acid (96/102/2), at a flow rate of $1.0 \mathrm{~mL} / \mathrm{min}$ in isocratic mode. The retention time of the OTA was about $5.5 \mathrm{~min}$.

\subsubsection{CTN Prodution by Medicinal Herbs}

Five grams of samples was mixed with $25 \mathrm{~mL}$ methanol-water (70/30) and homogenated at high speed for $2 \mathrm{~min}$, and then filtered using quantitative filter paper. One milliliters of the filtrate was diluted with $49 \mathrm{~mL}$ of phosphoric acid solution $(10 \mathrm{mmol} / \mathrm{L})$ and filtered through a glass fiber filter paper. The filtrate was purified by Citrinin Immunoaffinity Column (CitriTest HPLC, 3 mL, VICAM, Milford, MA, USA). The extract was eluted with $1.0 \mathrm{~mL}$ methanol-phosphoric acid solution (70/30) at a rate of 1-2 drops/s and then evaporated to dryness under a stream of nitrogen and analyzed using HPLC. HPLC analysis was performed with LC-20AT coupled to a FLD detector set to 350 nm excitation and $500 \mathrm{~nm}$ emission. The sample was separated using a ZORBAX SB C18 column $(150 \times 4.6 \mathrm{~mm}$, 5-Micron, Agilent Technologies Co., Ltd., Santa Clara, CA, USA). The mobile phase consisted of (A)

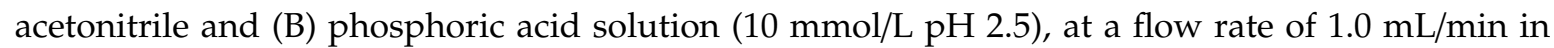
gradient mode, $0-10 \mathrm{~min}: 20 \%$ of $\mathrm{A}, 11-14 \mathrm{~min}$ : $20 \%-70 \%$ of $\mathrm{A}, 14-18 \mathrm{~min}: 70 \%-20 \%$ of $\mathrm{A}, 18-20 \mathrm{~min}$ : $20 \%$ of $\mathrm{A}$. The retention time of the CTN was about $7.5 \mathrm{~min}$.

\subsection{Isolation and Purification}

Ten grams of samples was added to $90 \mathrm{~mL}$ of sterile $0.85 \%$ physiological saline and mixed to prepare a 1:10 test solution [27]. Serial decimal dilutions were obtained and cultured on Sabouraud dextrose agar (SDA, $10 \mathrm{~g}$ of peptone, $40 \mathrm{~g}$ of dextrose, $15 \mathrm{~g}$ of agar, $0.1 \mathrm{~g}$ chloramphenicol, and disosslved with to $1 \mathrm{~L}$ with distilled water) [31] at $25^{\circ} \mathrm{C}$ for $5-7 \mathrm{~d}$, and the colony morphology was observed and recorded. For uncut samples, the surface of the sample was directly scraped off with a sterile inoculating loop and inoculated onto SDA or malt extract agar medium (MEA, $130 \mathrm{~g}$ of malt extract, $15 \mathrm{~g}$ of agar, $0.1 \mathrm{~g}$ chloramphenicol, and dissolved with $1 \mathrm{~L}$ with distilled water), and the mildewed parts were primarily scraped off. After incubation at $25^{\circ} \mathrm{C}$ for $5-7 \mathrm{~d}$, colony morphology was observed and recorded. Colonies with different morphological characteristics were picked and inoculated on SDA, separated, and purified to obtain pure cultures, and colony morphology and microbial growth in the medium were observed.

\subsection{Phenotypic Characterization}

For macromorphological observations, SDA, MEA, and Czapek Yeast Exatract Agar (CYA, $5 \mathrm{~g}$ yeast extract, $3 \mathrm{~g}$ of $\mathrm{NaNO}_{3}, 1 \mathrm{~g}$ of $\mathrm{K}_{2} \mathrm{HPO}_{4}, 0.5 \mathrm{~g} \mathrm{KCL}, 0.5 \mathrm{~g} \mathrm{MgSO} \cdot 7 \mathrm{H}_{2} \mathrm{O}, 0.01 \mathrm{~g} \mathrm{FeSO} \cdot 7 \mathrm{H}_{2} \mathrm{O}, 30 \mathrm{~g}$ sucrose, $15 \mathrm{~g}$ of agar, and disosslved with $1 \mathrm{~L}$ with distilled water) was used. Isolates were inoculated 
at one or three points on each plate of each medium and incubated at $25{ }^{\circ} \mathrm{C}$ in the dark for $3-7 \mathrm{~d}$. For microscopic morphological observations by biological microscope CX21FS1C (Olympus Ltd., Guangzhou, China), microscopic mounts were carried out in lactic acid from colonies cultured on MEA, and a drop of ethanol was added to eliminate air bubbles and excess conidia [8].

\subsection{DNA Extraction, Sequencing, and Analysis}

DNA was extracted from the cells, using the E.Z.N.A. ${ }^{\circledR}$ fungal DNA mini kit (Omega Bio-tek, Norcross, GA, USA) in accordance with the manufacturer's instructions. Three gene regions of the genomic DNA were amplified and sequenced: (1) the benA-specific primer pair Ben2f/Bt2b [32] was used to amplify the $\beta$-tublin gene. (2) The calmodulin gene was amplified using the CF1L/CF4 [33] primer pair. (3) The internally transcribed spacer (ITS) region was amplified using the ITS1/ITS4 primer pair [34]. Sequencing analysis was performed at BGI Co. Ltd. (Guangzhou, China) and the sequencing results were analyzed using BLAST by searching the NCBI nucleotide database for the genus and species of the isolates.

\subsection{Qualitative Determination of Mycotoxin-Producing Strains}

Thirty-seven strains (including 18 strains of Aspergillus, 17 strains of Penicillium, and 2 strains of Byssochlamys) were used to determine the presence of $\mathrm{AFB}_{1}, \mathrm{AFB}_{2}, \mathrm{AFG}_{1}, \mathrm{AFG}_{2}, \mathrm{CTN}$, and OTA. Among the total isolates obtained from the samples, 37 strains potentially producing six mycotoxins were cultured in $200 \mathrm{~mL}$ Sabouraud dextrose medium (SD, $10 \mathrm{~g}$ of peptone, $40 \mathrm{~g}$ of dextrose, $0.1 \mathrm{~g}$ chloramphenicol, and disosslved with $1 \mathrm{~L}$ with distilled water) at $25^{\circ} \mathrm{C}$ and $200 \mathrm{rpm}$ for $5 \mathrm{~d}$. Furthermore, standard strains Penicillium viridicatum Westling AS 3.4517 and Aspergillus parasiticus Speare AS 3.124 were used as the positive control. Penicillium viridicatum reportedly produces CTN [35]; Aspergillus parasiticus, $\mathrm{AFB}_{1}, \mathrm{AFB}_{2}, \mathrm{AFG}_{1}$, and $\mathrm{AFG}_{2}[20]$.

All liquid cultures of strains were sterilized by IRM G65 autoclaves (Frankfurk, Germany). The sterilized liquid cultures were ultrasonically extracted for $30 \mathrm{~min}$, vortex-mixed for $10 \mathrm{~min}$, and then transferred to a 50-mL centrifuge tube, and centrifuged at $3500 \mathrm{rpm}$ for $5 \mathrm{~min}$, and the supernatant was purified using the quantitative filter paper and transferred to another centrifuge tube. To $10 \mathrm{~mL}$ of extract, $20 \mathrm{~mL}$ of PBS was added, followed by immunoaffinity column chromatography, as previously reported [21]. The flow rate was maintained at $1-3 \mathrm{~mL} / \mathrm{min}$. The immunoaffinity column was rinsed with $10 \mathrm{~mL}$ PBS buffer and $1 \mathrm{~mL}$ of methanol. In three independent samples, and the eluate was blow-dried at $55^{\circ} \mathrm{C}$ with nitrogen, and the residue was dissolved in $1 \mathrm{~mL}$ of acetonitrile:water (1:1), vortex-mixed for $30 \mathrm{~s}$, passed through a $0.22-\mu \mathrm{m}$ microporous membrane, and harvested for injection. HPLC-MS/MS analysis was performed with a 1290 Infinity Liquid Chromatograph interfaced to a 6430 Triple Quad Mass Spectrometer system. The sample was separated using a Hypersil Gold C18 column $(2.1 \times 100 \mathrm{~mm}$, 3-Micron). The mobile phase A: aqueous containing 1\% formic acid, mobile phase B: acetonitrile; programmed gradient elution, flow rate: $0.3 \mathrm{~mL} / \mathrm{min}$; column temperature: $35^{\circ} \mathrm{C}$; injection volume: $5 \mu \mathrm{L}$. The mass spectrometer was operated in the positive mode with multiple reaction monitoring $(\mathrm{MRM})$. Two precursor-to-product ion transitions were simultaneously monitored at $m / z$ 313.0-285.0, $m / z$ 313.0-269.0 for $\mathrm{AFB}_{1} ; m / z$ 315.0-287.1, $m / z$ 315.0-259.1 for $\mathrm{AFB}_{2} ; m / z$ 329.1-243.2, $m / z$ 329.1-311.0 for $\mathrm{AFG}_{1} ; m / z$ 331.0-313.0, $m / z$ 331.0-245.0 for $\mathrm{AFG}_{2} ; m / z$ 404.1-239.0, $m / z$ 404.1-358.0 for OTA; and $m / z$ 251.0-233.0, $m / z$ 251.0-205.0 for CTN (Figure S2).

\subsection{Multiplex PCR}

To optimize the multiplex PCR assay to detect AF-producing fungal species in samples of medicinal herbs on the basis of genes [36,37] involved in mycotoxin biosynthesis (Table 2), reference fungal strains were confirmed via monoplex PCR. The multiplex PCR was standardized by empirically varying critical factors affect multiplexing, such as primer concentrations, template concentration, and annealing temperature. The PCR conditions were as follows: initial heat activation of DNA polymerase at $94{ }^{\circ} \mathrm{C}$ for $2 \mathrm{~min}$, followed by 35 cycles of denaturation at $94{ }^{\circ} \mathrm{C}$ for $30 \mathrm{~s}$, annealing at 
$55{ }^{\circ} \mathrm{C}$ for $30 \mathrm{~s}$, extension at $72{ }^{\circ} \mathrm{C}$ for $90 \mathrm{~s}$, and a final extension at $72{ }^{\circ} \mathrm{C}$ for $7 \mathrm{~min}$. PCR products were electrophoresed on a 1.6\% agarose gel with a 1000-bp DNA size marker at $100 \mathrm{~V}$ for $25 \mathrm{~min}$.

\subsection{Statistical Analysis}

All assays were replicated three times for each treatment. We used the websites http://www. ncbi.nlm.nih.gov/ for blast research at the GenBank database. The two-way sequencing results were spliced by DANMAN Version 8 (Lynon Corporation, SanRamon, CA, USA) and used MEGA Version 7.0.26 (The Pennsylvania State University, University Park, PA, USA) to analyze sequence variability, calculate genetic distance, and construct the phylogenetic tree [38]. The evolutionary distances were computed using the Maximum Composite Likelihood method [39] and are in the units of the number of base substitutions per site.

Supplementary Materials: Supplementary materials can be accessed at: http:/www.mdpi.com/2072-6651/12/1/30/s1, Figure S1: HPLC-FLD chromatograms for $(\mathrm{A}) \mathrm{AFB}_{1}, \mathrm{AFB}_{2}, \mathrm{AFG}_{1}, \mathrm{AFG}_{2}$ standard $\left(\mathrm{AFB}_{1}, \mathrm{AFG}_{1}=10 \mathrm{ng} \mathrm{mL}{ }^{-1}\right.$; $\left.\mathrm{AFB}_{2}, \mathrm{AFG}_{2}=3 \mathrm{ng} \mathrm{mL}{ }^{-1}\right) ;(\mathbf{B}) \mathrm{AFB}_{1}, \mathrm{AFB}_{2}, \mathrm{AFG}_{2}$ positive samples (Ganderma lucidum 06-3); (C) OTA standard $\left(\mathrm{OTA}=20 \mathrm{ng} \mathrm{mL}^{-1}\right)$; (D) OTA positive samples (Codonopsis radix 03-1); (E) CTN standard $\left(\mathrm{CTN}=10 \mathrm{ng} \mathrm{mL}^{-1}\right)$; $(\mathbf{F})$ CTN positive samples (Tremella fuciformis 10-3). Figure S2. HPLC-MS/MS chromatograms with MRM modes for (A) $\mathrm{AFB}_{1}, \mathrm{AFB}_{2}, \mathrm{AFG}_{1}, \mathrm{AFG}_{2}$ standard; (B) OTA standard; (C) $\mathrm{CTN}$ standard; (D) $\mathrm{AFB}_{1}, \mathrm{AFB}_{2}$ positive strain (Aspergillus flavus isolated from Amomi fructus 08-3); (E) CTN positive strain (Aspergillus chevalieri isolated from Amomi fructus 08-1). Two precursor-to-product ion transitions were simultaneously monitored at $\mathrm{m} / \mathrm{z} 313.0-285.0$, $m / z$ 313.0-269.0 for $\mathrm{AFB}_{1} ; m / z$ 315.0-287.1, $m / z$ 315.0-259.1 for $\mathrm{AFB}_{2} ; \mathrm{m} / \mathrm{z} 329.1-243.2, \mathrm{~m} / \mathrm{z} 329.1-311.0$ for $\mathrm{AFG}_{1}$; $\mathrm{m} / \mathrm{z}$ 331.0-313.0, $\mathrm{m} / \mathrm{z}$ 331.0-245.0 for $\mathrm{AFG}_{2} ; \mathrm{m} / \mathrm{z} 404.1-239.0, \mathrm{~m} / \mathrm{z} 404.1-358.0$ for OTA; and $\mathrm{m} / \mathrm{z} 251.0-233.0, \mathrm{~m} / \mathrm{z}$ 251.0-205.0 for CTN. Table S1: Calibration curve, limit of quantification and limit of detection of six mycotoxins.

Author Contributions: Conceived and designed the experiments, L.C., W.G., Q.W., and J.Z.; Performed the experiments, L.C., Y.Z., J.Z., T.L., D.L., M.Z., and Y.Z.; Analyzed the data, L.C., W.G., and W.C.; Wrote and revised the paper, L.C. All authors have read and agreed to the published version of the manuscript.

Funding: This work was supported by the National Key R\&D Program of China (2018YFD0400901) and GDAS' Project of Science and Technology Development (2019GDASYL-0201001).

Conflicts of Interest: The authors declare no conflict of interest.

\section{References}

1. Liu, C.M.; Qin, J.A.; Dou, X.W.; Yang, M.H.; Sun, X.B. Extrinsic harmful residues in Chinese herbal medicines: Types, detection, and safety evaluation. Chin. Herb. Med. 2018, 10, 117-136. [CrossRef]

2. Chen, X.P. Analysis on the problems of 3477 batches Chinese herbal pieces by our hospital acceptance. China Med. Her. 2014, 11, 139-142. [CrossRef]

3. Liu, Y.P.; Ma, H.C.; Zeng, Q.Z. Quality evaluation of 733 batches of Chinese herbal medicines. World Latest Med. Inf. 2019, 19, 238-239.

4. Chen, J.; Gao, W.W.; Tang, D.; Cai, F.; Yang, M.H. Investigation of fungal populations in seven ochratoxin A contaminated root herbs. China J. Chin. Mater. Med. 2010, 35, 2647-2651.

5. Shi, Q.N. Evaluation of Moldy Fungus and Mycotoxin Contamination in Coix Seed. Master's Thesis, Guizhou University, Guizhou, China, June 2017.

6. Han, Z.; Ren, Y.P.; Zhu, J.F.; Cai, Z.X.; Chen, Y.; Luan, L.J.; Wu, Y.J. Multianalysis of 35 mycotoxins in traditional Chinese medicines by ultra-high-performance liquid chromatography-tandem mass spectrometry coupled with accelerated solvent extraction. J. Agric. Food Chem. 2012, 60, 8233-8247. [CrossRef] [PubMed]

7. Abramson, D.; Mills, J.T.; Marquardt, R.R.; Frohlich, A.A. Mycotoxins in fungal contaminated samples of animal feed from western Canada, 1982-1994. Can. J. Vet. Res. 1997, 61, 49-52.

8. Frisvad, J.C.; Hubka, V.; Ezekiel, C.N.; Hong, S.B.; Nováková, A.; Chen, A.J.; Arzanlou, M.; Larsen, T.O.; Sklenáŕ, F; Mahakarnchanakul, W.; et al. Taxonomy of section and their production of aflatoxins, ochratoxins and other mycotoxins. Stud. Mycol. 2019, 93, 1-63. [CrossRef]

9. Hou, L.L.; Zhou, X.; Gan, F.; Liu, Z.X.; Zhou, Y.J.; Qian, G.; Huang, K. Combination of selenomethionine and $\mathrm{N}$-acetylcysteine alleviates the joint toxicities of aflatoxin $\mathrm{B} 1$ and ochratoxin A by ERK MAPK signal pathway in porcine alveolar macrophages. J. Agric. Food Chem. 2018, 66, 5913-5923. [CrossRef] 
10. Zhang, L.; Dou, X.W.; Zhang, C.; Logrieco, A.F.; Yang, M.H. A review of current methods for analysis of mycotoxins in herbal medicines. Toxins 2018, 10, 65. [CrossRef]

11. Zain, M.E. Impact of mycotoxins on humans and animals. J. Saudi Chem. Soc. 2011, 15, 129-144. [CrossRef]

12. Yang, L.; Wang, L.N.; Pan, J.Y.; Xiang, L.; Yang, M.H.; Logrieco, A.F. Determination of ochratoxin A in traditional Chinese medicinal plants by HPLC-FLD. Food Addit. Contam. Part A Chem. Anal. Control Expo. Risk Assess. 2010, 27, 989-997. [CrossRef] [PubMed]

13. International Agency for Research on Cancer. IARC Monographs on the Evaluation of Carcinogenic Risks to Humans; International Agency for Research on Cancer: Lyon, France, 1993; Volume 56.

14. JECFA (Joint FAO/WHO Expert Committee on Food Additives). Safety Evaluation of Certain Mycotoxins in Food; WHO Food Additives Series 47; FAO Food and Nutrition Paper 74. Available online: http: //www.inchem.org/documents/jecfa/jecmono/v47je01.htm (accessed on 6 February 2001).

15. Scientific Committee on Food. Opinion of the Scientific Committee on Food on Ochratoxin A; European Commission: Brussels, Belgium, 1998.

16. Mandeel, Q.A. Fungal contamination of some imported spices. Mycopathologia 2005, 159, 291-298. [CrossRef]

17. Sudharsan, S.; Malka, B.; Varda, Z.; Moshe, K.; Anatoly, T.; Elazar, Q.; Edward, S. Rapid detection and identification of mycotoxigenic fungi and mycotoxins in stored wheat grain. Toxins 2017, 9, 302.

18. Zheng, R.S.; Wang, W.L.; Tan, J.; Xu, H.; Zhan, R.T.; Chen, W.W. An investigation of fungal contamination on the surface of medicinal herbs in China. Chin. Med. 2017, 12, 2. [CrossRef] [PubMed]

19. Ayanbimpe, G.M.; Ojo, T.K.; Afolabi, E.; Opara, F.; Orsaah, S.; Ojerinde, O.S. Evaluation of extracts of Jatropha curcas and Moringa oleifera in culture media for selective inhibition of saprophytic fungal contaminants. J. Clin. Lab. Anal. 2009, 23, 161-164. [CrossRef] [PubMed]

20. Bhatnagar, D.; McCormick, S.P.; Lee, L.S.; Hill, R.A. Identification of O-methylsterigmatocystin as an aflatoxin B1 and G1 precursor in Aspergillus parasiticus. Appl. Environ. Microbiol. 1987, 53, 1028-1033. [CrossRef] [PubMed]

21. Tassaneeyakul, W.; Razzazi-Fazeli, E.; Porasuphatana, S.; Bohm, J. Contamination of aflatoxins in herbal medicinal products in Thailand. Mycopathologia 2004, 158, 239-244. [CrossRef]

22. Yang, H.; Wang, X.F.; Li, Z.J.; Guo, Q.B.; Yang, M.G.; Chen, D.; Wang, C.L. The effect of blue light on the production of citrinin in M9 by regulating the gene through lncRNA. Toxins 2019, 11, 536. [CrossRef]

23. Li, Y.P.; Pan, Y.F.; Zou, L.H.; Xu, Y.; Huang, Z.B.; He, Q.H. Lower citrinin production by gene disruption of ctnB involved in citrinin biosynthesis in Monascus aurantiacus Li AS3.4384. J. Agric. Food Chem. 2013, 61, 7397-7402. [CrossRef]

24. Salah, A.; Bouaziz, C.; Prola, A.; Pires, D.S.J.; Bacha, H.; Abid-Essefi, S.; Lemaire, C. Citrinin induces apoptosis in human HCT116 colon cancer cells through endoplasmic reticulum stress. J. Toxicol. Environ. Health Part A 2017, 80, 1230-1241. [CrossRef]

25. Gong, L.; Zhu, H.; Li, T.T.; Ming, G.F.; Duan, X.W.; Wang, J.S.; Jiang, Y.M. Molecular signatures of cytotoxic effects in human embryonic kidney 293cells treated with single and mixture of ochratoxin A and citrinin. Food Chem. Toxicol. 2019, 123, 374-384. [CrossRef] [PubMed]

26. El-Kady, I.; El-Maraghy, S.; Zohri, A.N. Mycotoxin producing potential of some isolates of Aspergillus flavus and eurotium groups from meat products. Microbiol. Res. 1994, 149, 297-307. [CrossRef]

27. Chinese Pharmacopoeia Commission. Pharmacopoeia of the People's Republic of China; Chinese Medicinal Science and Technology Press: Beijing, China, 2015.

28. National Health Commission of the People's Republic of China, China Food and Drug Adnubustratuin. Determination of Aflatoxin B and G in Foods in National Food Safety Standards: GB 5009.22-2016; China Standard Press: Beijing, China, 2016.

29. National Health Commission of the People's Republic of China, China Food and Drug Adnubustratuin. Determination of Ochratoxin A in Foods of National Food Safety Standards: GB 5009.96-2016; China Standard Press: Beijing, China, 2016.

30. National Health Commission of the People's Republic of China, China Food and Drug Adnubustratuin. Determination of Citrinin in Foods of National Food Safety Standards: GB 5009.222-2016; China Standard Press: Beijing, China, 2016.

31. Scognamiglio, T.; Zinchuk, R.; Gumpeni, P.; Larone, D.H. Comparison of inhibitory mold agar to Sabouraud dextrose agar as a primary medium for isolation of fungi. J. Clin. Microbiol. 2010, 48, 1924-1925. [CrossRef] [PubMed] 
32. Samson, R.A.; Houbraken, J.A.M.P.; Kuijpers, A.F.A. New ochratoxin A or sclerotium producing species in Aspergillus section Nigri. Stud. Mycol. 2004, 50, 45-61.

33. Peterson, S.W. Phylogenetic analysis of Aspergillus species using DNA sequences from four loci. Mycologia 2008, 100, 205-226. [CrossRef] [PubMed]

34. White, T.J. Amplification and direct sequencing of fungal ribosomal RNA genes for phylogenetics. PCR Protoc. A Guide Methods Appl. 1990, 18, 315-322.

35. Krogh, P.; Hasselager, E.; Friis, P. Studies on fungal nephrotoxicity: 2. Isolation of two nephrotoxic compounds from Penicillium viridicatum Westling: Citrinin and oxalic acid. Acta Pathol. Microbiol. Scand. B Microbiol. Immunol. 1970, 78, 401-413. [CrossRef]

36. People's Republic of China, State Administration of Quality Supervision, Inspection and Quarantine. Detection of Aflatoxigenic Strain of Aspergillus by PCR: SN/T 2582-2010; China Standard Press: Beijing, China, 2010.

37. Priyanka, S.R.; Venkataramana, M.; Kumar, G.P.; Rao, V.K.; Murali, H.C.S.; Batra, H.V. Occurrence and molecular detection of toxigenic Aspergillus species in food grain samples from India. J. Sci. Food Agric. 2014, 94, 537-543. [CrossRef]

38. Kumar, S.; Stecher, G.; Tamura, K. MEGA7: Molecular Evolutionary Genetics Analysis Version 7.0 for Bigger Datasets. Mol. Biol. Evol. 2016, 33, 1870-1874. [CrossRef]

39. Tamura, K.; Nei, M.; Kumar, S. Prospects for inferring very large phylogenies by using the neighbor-joining method. Proc. Natl. Acad. Sci. USA 2004, 101, 11030-11035. [CrossRef]

(C) 2020 by the authors. Licensee MDPI, Basel, Switzerland. This article is an open access article distributed under the terms and conditions of the Creative Commons Attribution (CC BY) license (http://creativecommons.org/licenses/by/4.0/). 